\title{
Pertinent Issues in Diagnosis and Management of Wheezing in Under-five Children at Community Level
}

\author{
Dheeraj Shah and Piyush Gupta \\ From the Department of Pediatrics, University College of Medical Sciences (University of Delhi) and GTB Hospital, Delhi 110 095, \\ India. \\ Correspondence to: Dr Dheeraj Shah, Associate Professor, Department of Pediatrics, University College of Medical Sciences and \\ GTB Hospital, Dilshad Garden, Delhi 110 095, India. shahdheeraj@hotmail.com
}

\begin{abstract}
Wheezing in acute respiratory infections is a rule rather than exception. A large proportion of children (up to $75 \%$ ) having 'pneumonia' or 'severe pneumonia' as per WHO definitions have associated wheezing. The current strategies to diagnose and manage wheeze in the community need to be updated, as audible wheeze is present in only less than one-third of wheezy children, and can not be relied upon solely. A history of previous episodes of respiratory distress has a high sensitivity to diagnose wheezy disorders. In a significant proportion of children, the respiratory rate comes back to normal and the chest indrawing disappear after two to three cycles of inhaled bronchodilator medications. Operational research is needed to evaluate the feasibility of including management of wheezing in the community based ARI management programs.
\end{abstract}

Keywords: Pheumonia, Respiratory tract infections, Wheezing.

\section{W} heezing is associated with or contributory to a large proportion of childhood acute respiratory infections (ARI). For logistic reasons, the World Health Organization (WHO) strategy for the control of ARI has focused on the case management of pneumonia in an attempt to reduce mortality. In the last two decades, researchers from developing countries have raised serious concerns over the applicability of these guidelines in children having wheeze. The diagnosis and management of wheeze has largely been ignored resulting in overuse of antibiotics and underuse of bronchodilator medications at the community level. The operational aspects and advocacy related to management of wheeze in ARI are also missing from most health programs in developing countries.To rationalize the antimicrobial and bronchodilator prescription, it is essential to make a reasonably accurate diagnosis of wheeze using simple clinical tools.

INDIAN PEDIATRICS

\section{How Common is WheEzing In ARI?}

Wheezing is a musical sound produced when the air flow from the lungs is obstructed, due to contraction of the smooth muscles surrounding the airways or swelling of the lining of bronchioles. The main causes of wheezing in under-five children are bronchial asthma, bronchiolitis and pneumonia. Inhaled foreign bodies, compression of the airways, from outside (as by lymph nodes or a tumour), and pulmonary edema are less common.

Wheezing is a very common symptom or sign in under-five children, including infants. Children with wheeze can have fast breathing or/and lower chest indrawing, and are likely to be categorized as pneumonia or severe pneumonia according to the current ARI case management guidelines. Hospital based studies from Delhi, India have documented asthma to be more common diagnosis than pneumonia in under-five children having the symptoms of cough or difficult breathing(1-3). Using 
the WHO/IMCI algorithms, there was a considerable overdiagnosis of pneumonia because of inclusion of cases of asthma and other respiratory ailments $(1,2)$. In a study enrolling children aged between six months to five years, wheezing was found in $75 \%$ (150 out of 200) of the subjects presenting with signs of fast breathing or lower chest indrawing(3). Asthma was the predominant diagnosis in almost half of these cases whereas pneumonia (with or without wheezing) was the diagnosis in only one-third of cases. In an Indian multicentric study designed for comparing the efficacy of three day and five day amoxicillin treatment in non-severe pneumonia, wheezing was present in 13\% of cases(4). The low prevalence of wheezing in this study was because of a two stage screening procedure resulting in exclusion of children with recurrent respiratory distress, and also those in whom 'fast breathing' disappeared after an initial course of bronchodilators. In another recent multicentric study enrolling more than 2000 underfive children from rural primary health centers in India, wheezing was documented in $22 \%$ of children having WHO defined non-severe pneumonia, despite excluding cases with recurrent respiratory distress(5). In children having lower chest indrawing (categorized as severe pneumonia as per WHO definition), the prevalence of wheezing is even higher. In a recent multicentric trial evaluating the efficacy of oral drugs in treatment of severe pneumonia in children from Pakistan(6), wheezing on auscultation was present in $76 \%$ of 2037 children with lower chest indrawing despite excluding known asthmatics and those responding to initial bronchodilators. The prevalence of wheezing was much more in infants in comparison to children aged more than one year ( $82 \%$ vs. 65\%).

From the foregoing, it is evident that wheezing is present in majority of children diagnosed as pneumonia or severe pneumonia, based on the presence of fast breathing and chest indrawing, respectively. Wheezing is common even in those children who are not having recurrent episodes of respiratory distress, and those who do not respond to initial course of inhaled bronchodilator therapy.

\section{Diagnosis of Wheeze in the Community}

The current WHO strategy for the management of
ARI relies heavily on standardized case management for preventing pneumonia deaths. For management of wheeze, the guidelines recommend giving two cycles of rapid acting inhaled bronchodilator at 15 minute intervals to children with audible wheeze and fast breathing and/or lower chest indrawing. Thus, the diagnosis of wheeze is made by the health worker only if the child has audible wheeze. Majority of children with wheezing do not get identified by health workers using this approach as only less than one-third of the children with auscultatory wheeze have audible wheeze. In a study from Delhi hospital, audible wheeze was appreciated in only 44 of the 150 cases (29.3\%) with an auscultable wheeze(3). In a recent multicentric study evaluating the role of antibiotics in treatment of wheezy pneumonia in under-five Indian children, audible wheeze was found in only $17 \%$ of cases where it was present on auscultation(7). The multicentric study enrolling children with severe pneumonia from Pakistan also documented a prevalence of audible wheeze in only $17 \%$ (261 out of 1545) of the total wheezy children(6). The later two studies had a lower prevalence of audible wheezing in comparison to the earlier data from India $(1,3)$ due to exclusion of cases with known asthma and recurrent episodes of respiratory distress. In another multicentric study from Pakistan enrolling all wheezy children, the prevalence of audible wheeze was 37\%(8).

As the present strategy of relying on audible wheeze for institution of bronchodilator therapy results in gross underutilization of bronchodilators and overprescription of antibiotics, attempts have been made to refine the community based diagnosis of wheezing. In an observational study from New Delhi, India(1), the best predictor for asthma related wheeze was two or more earlier similar episodes (sensitivity $84 \%$, specificity $84 \%$ ) followed by presence of fever (sensitivity $73 \%$ and specificity $84 \%$ ). It was further suggested to include these simple clinical features [history of: (i) previous similar episode of cough and difficult breathing, and (ii) fever] in the WHO case management algorithm to significantly refine the antibiotic and bronchodilator prescription. The alternative algorithm represented a significant improvement over the WHO algorithm, primarily by restricting over-prescription of the antibiotics and under-utiliza- 
tion of the bronchodilators. Another option is to give therapeutic trial with inhaled bronchodilators before assigning the diagnosis of pneumonia or severe pneumonia in all children with 'fast breathing' or 'chest indrawing.' Among cases of non-severe pneumonia and wheeze, the respiratory rate is known to come back below age specific cut-offs in 46\%-62\% children $(7,8)$. The response rate in children with lower chest indrawing is somewhat lower(8). However, such an approach is likely to result in considerable overuse of bronchodilator drugs, and may even delay the timely management or referral of children with non-wheezy illnesses, especially in those with lower chest indrawing. The best approach remains the appropriate diagnosis of wheeze by skilled personnel using the stethoscope. In settings with high prevalence of wheeze, the possibility of training health workers in the use of the stethoscope should be explored in order to further rationalize the case management of children presenting with cough or difficult breathing. The first referral units need to be equipped for managing wheezy disorders.

\section{TreatMenT OF WHEEZE IN CHILDREN WITH ACUTE RESPIRATORY INFECTIONS}

WHO recommended case management of children presenting with cough or difficult breathing, is structured towards treatment as pneumonia in preference to acute asthma. With changing disease epidemiology and rapid urbanization, the prevalence of wheezy disorders, including asthma is increasing in all age groups. Accurate diagnosis and early institution of bronchodilator therapy is important to prevent asthma related fatalities.

\section{Antibiotics}

Antibiotics have no role in management of wheezing due to bronchial asthma. A history of recurrent episodes of respiratory distress in the past, and good response to initial bronchodilator therapy have high specificity to diagnose asthma in children presenting with 'fast breathing' or 'chest indrawing.' It is worthwhile to withhold antibiotic therapy in such situations, resulting in a substantial reduction in prescription of antibiotics.

Although asthma is the most common cause, infections of the respiratory tract are also well known causes of wheezing and acute exacerbation of asthma in children. Viruses are the most common cause of wheezing associated with respiratory tract infections. Respiratory syncytial virus (RSV) infections have long been recognized to produce the first episode of wheezing in children, some of whom go on to develop chronic asthma(9). The risk of pneumonia or bronchiolitis caused by RSV is highest among children aged less than 2 years with the most severe disease occurring in infants aged 3 weeks to 3 months. The rhinovirus, adenovirus and human bocaviruses are other viruses responsible for wheezing in children hospitalized because of respiratory infection(10). Antibiotics have no role in treatment of bronchiolitis due to RSV infection(11). Though most literature suggest viruses as the predominant cause of respiratory infection associated wheezing, there is some evidence suggesting a high prevalence of wheezing amongst children having pneumonia due to Streptococcus pneumoniae and Haemophilus influenzae, the two most common bacteria responsible for pneumonia(12). Viral or bacterial coinfection is also a common finding in young children with pneumonia in developing countries(12-14). Overall, clinical differentiation of viral and bacterial etiology in under-five children persisting to have wheeze after initial bronchodilator therapy, is difficult in community settings.

A multi-centric, double blind randomized placebo-controlled trial was conducted in outpatient departments of 8 referral hospitals in India(7), to specifically assess whether children of non-severe pneumonia with wheeze can be effectively managed without antibiotics and to identify which sub-group of cases do require antibiotics. Clinical failures occurred in 201 out of 836 cases (24.0\%) on placebo and 166 out of 835 cases (19.9\%) on amoxicillin (risk difference $4.2 \%$ in favour of antibiotic, 95\% CI: 0.2 to 8.1). Clinical failure was associated with placebo treatment (adjusted OR=1.28, 95\% CI: 1.01 to 1.62 ). The trial concluded that treating children with non-severe pneumonia and wheeze with a placebo is not equivalent to treatment with oral amoxicillin. However, the difference in failure rate between two groups was less than $5 \%$ in both 'per protocol analysis' and 'intention-to-treat analysis.'

On the basis of available evidence, it appears 
justified to continue the practice of giving antibiotics to children who are diagnosed in the community to be having wheeze associated with pneumonia, and who do not respond to initial bronchodilator therapy. The choice of antimicrobial in such situation is similar to that in other children with pneumonia.

\section{Bronchodilator Medications}

As there is substantial evidence that wheeze is common in children fitting into the criteria of either 'pneumonia' or 'severe pneumonia' as per WHO/ IMCI guidelines, and also that these signs are likely to disappear after two to three cycles of inhaled bronchodilator therapy in about one-third to half of the cases, this form of therapy has a major role in management of children presenting with cough or difficult breathing. Inhaled beta- 2 adrenergic drugs (salbutamol or terbutaline) remain the mainstay of bronchodilator therapy for treatment of wheeze. These drugs can be given in young children by either nebulizer or metered dose inhalers (MDI) along with spacer and mask attachment. Delivery of bronchodilator medications via a nebulizer driven by an electric compressor or oxygen cylinder is an effective, safe and well-established technique. However, need of electricity to run the compressor, constraints in availability of high flow oxygen cylinders, and high cost of equipments limit the routine use of this technique in most primary health care settings of developing countries. Metered dose inhalers with spacer devices may be the most appropriate method for administering these medications to children in these settings, due to their easy availability and ease of administration. There is substantial evidence showing the efficacy of such devices in treatment of childhood asthma. The cost of equipments can further be reduced by using improvised home made spacer devices. A systematic review(15) of six trials comparing efficacy of commercial and home-made spacers in childhood asthma did not demonstrate any significant difference between the two delivery methods in terms of the need for hospital admission (RR 1.00, 95\% CI 0.63 to 1.59), change in oxygen saturation (SMD -0.03, 95\% CI -0.39 to 0.33), peak expiratory flow rate (SMD 0.04, 95\% CI -0.72 to 0.80), clinical score (WMD $0.00,95 \%$ CI -0.37 to 0.37 ), or need for additional treatment (RR 1.18, 95\% CI 0.84 to 1.65).
Despite high prevalence and possibility of successful management using simple tools and medications, published evidence regarding management of wheeze in the community in a developing country setting is severely lacking. The subsequent clinical course of children who respond to initial bronchodilator therapy, and are sent home without antibiotics, is also a case of concern. A multicentric study from Pakistan designed to study the course and profile of under-five children managed with 3 cycles of inhaled bronchodilator therapy, documented that $15 \%$ and $38 \%$ of the non-severe and severe pneumonia group, respectively showed subsequent deterioration on follow up(8). No family history of wheeze, fever (temperature $>100^{\circ} \mathrm{F}$ ), and lower chest indrawing were identified as predictors of subsequent deterioration. It is, therefore, prudent to ensure adequate follow-up of children diagnosed and managed in the community with initial course of inhaled bronchodilators. The issues related to safety and adherence to the continuation of bronchodilator medications at home also need to be addressed. The clinical outcome of under-five children with wheeze has been shown to be similar when treated with oral or inhaled salbutamol at home after initial response to inhaled bronchodilator therapy(16).

\section{EPILOGUE}

As wheezing is seen in majority of under-five children who are otherwise diagnosed as having 'pneumonia' or 'severe pneumonia' as per WHO/ IMCI definitions, the case management guidelines for diagnosis of wheeze in children with ARI need to be refined. A large proportion of these children can be successfully managed without antibiotics, using simple devices and medications. Health workers in community need to develop skills in recognizing and managing wheeze in order to successfully manage ARI, and to rationalize antibiotic therapy. Health care facilities managing children with ARI must be equipped with facilities to diagnose and treat wheezy children. Health programs involving management of childhood ARI in developing countries need to incorporate the diagnosis and management of wheeze. Operational research is urgently required to test the feasibility and utility of management of wheeze by trained health workers in actual field situations. 
Contributors: The article was conceived by PG and DS. DS searched the literature and wrote the paper, which was edited by PG. Both authors approved the final draft.

Competing Interests: None stated.

Funding: None.

\section{REFERENCES}

1. Sachdev HPS, Vasanthi B, Satyanarayana L, Puri RK. Simple predictors to differentiate acute asthma from ARI in children: implications for refining case management in the ARI Control Programme. Indian Pediatr 1995; 31: 1251-1259.

2. Shah D, Sachdev HPS. Evaluation of the WHO/ UNICEF algorithm for integrated management of childhood illness between the ages of two months to five years. Indian Pediatr 1999; 36: 767-777.

3. Sachdev HPS, Mahajan SC, Garg A. Improving antibiotic and bronchodilator prescription in children presenting with difficult breathing: Experience from an urban hospital in India. Indian Pediatr 2001; 38: 827-838

4. ISCAP Study Group. Three day versus five day treatment with amoxicillin for non-severe pneumonia in young children: a multicentre randomised controlled trial. BMJ 2004; 328: 791.

5. Awasthi S, Agarwal G, Singh JV, Kabra SK, Pillai RM, Singhi S, et al., for ICMR-IndiaClen Pneumonia Project Group. Effectiveness of 3-day amoxycillin vs. 5-day co-trimoxazole in the treatment of non-severe pneumonia in children aged 2-59 months of age: a multi-centric open labeled trial. J Trop Pediatr 2008; 54: 382-389.

6. Hazir T, Fox LM, Nisar YB, Fox MP, Ashraf YP, MacLeod WB, et al.; New Outpatient Short-Course Home Oral Therapy for Severe Pneumonia Study Group. Ambulatory short-course high-dose oral amoxicillin for treatment of severe pneumonia in children: a randomised equivalency trial. Lancet 2008; 371: 49-56.

7. Awasthi S, Agarwal G, Kabra SK, Singhi S, Kulkarni M, More V, et al. Does 3-day course of oral amoxycillin benefit children of non-severe pneumonia with wheeze: a multicentric randomised controlled trial. PLoS One 2008; 3: e1991.
8. Hazir T, Qazi S, Nisar YB, Ansari S, Maqbool S, Randhawa S, et al. Assessment and management of children aged 1-59 months presenting with wheeze, fast breathing, and/or lower chest indrawing; results of a multi centre descriptive study in Pakistan. Arch Dis Child 2004; 89: 1049-1054.

9. Pérez-Yarza EG, Moreno A, Lázaro P, Mejías A, Ramilo O. The association between respiratory syncytial virus infection and the development of childhood asthma: a systematic review of the literature. Pediatr Infect Dis J 2007; 26: 733-739.

10. Calvo C, García-García ML, Pozo F, Carvajal O, Pérez-Breña P, Casas I. Clinical characteristics of human bocavirus infections compared with other respiratory viruses in Spanish children. Pediatr Infect Dis J 2008; 27: 677-680.

11. Spurling GKP, Fonseka K, Doust J, Del MC. Antibiotics for bronchiolitis in children. Cochrane Database Syst Rev 2007; 1: CD005189.

12. Ghafoor A, Nomani NK, Ishaq Z, Zaidi SZ, Anwar F, Burney MI, et al. Diagnoses of acute lower respiratory tract infections in children in Rawalpindi and Islamabad, Pakistan. Rev Infect Dis 1990; 12 (Suppl 8); S907-S914.

13. Forgie IM, O’Neill KP, Lloyd-Evans N, Leinonen $\mathrm{M}$, Campbell H, Whittle HC, et al. Etiology of acute lower respiratory tract infections in Gambian children: I. Acute lower respiratory tract infections in infants presenting at the hospital. Pediatr Infect Dis J 1991; 10: 33-41.

14. Rudan I, Boschi-Pinto C, Biloglav Z, Mulholland $\mathrm{K}$, Campbell H. Epidemiology and etiology of childhood pneumonia. Bull World Health Organ 2008; 86: 408-416.

15. Rodriguez-Martinez CE, Sossa M, Lozano JM. Commercial versus home-made spacers in delivering bronchodilator therapy for acute therapy in children. Cochrane Database Syst Rev 2008; 2: CD005536.

16. Hazir T, Qazi S, Bin Nisar Y, Ansari S, Maqbool S, Randhawa S, et al. Comparison of clinical outcome with oral and inhaled bronchodilators in the management of wheezy children aged 1-59 months in the community: a randomized trial in Pakistan. Int J Tuberc Lung Dis 2004; 8: 1308-1314. 